\title{
Quantitative Assessment of TEM-Sample Warping Caused by FIB Preparation
}

\author{
R. Salzer, A. Graff, M. Simon, F. Altmann
}

Fraunhofer Institute for Mechanics of Materials, Walter-Huelse-Strasse 1, 06120 Halle Germany

Today focused ion beam technique (FIB) is well established and widely used in material science. One of its main applications is the preparation of samples for the transmission electron microscopy (TEM). For high resolution TEM investigations it is necessary to have samples with thicknesses in the range of only several $10 \mathrm{~nm}$, especially for high density materials. FIB-preparation of such thin lamellas can be extremely challenging due to warping of the TEM lamella (Fig. 1). Thus further thinning is very complicated or almost impossible.

To understand the fundamental mechanisms of the warping effect we have systematically investigated the influence of milling parameters, lamella area and thickness on the warping. Aim was to derive a reliable preparation regime which avoids the warping of the TEM sample. To determine the thickness of the TEM sample which is the basic geometric property a thickness measurement method basing on backscatter electron image correlation [1] is used. For the measurement of the sample deflection a laser scanning microscope (Fig. 2 a) is used. It was already shown, that any preparation with a focused ion beam leads to change of the mechanical properties at the irradiated surface $[2,3]$.

To estimate the initial stress induced by the focused ion beam molecular dynamic simulations of the FIB sputtering process on Silicon were performed. As a result it was predicted that at the interface region between the amorphisated layer and the undisturbed Si-crystal an initial compressive stress of $\sim 1 \mathrm{GPa}$ (Fig. 2 b) [4] occurs whilst $\mathrm{Ga}^{+}$irradiation. Additionally it could be shown that the thickness of this amorphous - crystalline interface region increases with increasing $\mathrm{Ga}^{+}$energy. It has to be mentioned that the MD simulations didn't take into account any stress compensation caused by recrysallisation or a deformation of a sample.

In order to understand the influence on the sample warping finite element simulations were performed using the information of the MD simulation about the stress state in the interface region and the geometry of a TEM lamella. As a result we obtained a similar morphology and a warping of the lamella comparable to that of real samples (Fig. 1, 3).

Based on these results we assume, that the deflection of thin TEM samples during FIB preparation is the equilibrium between the induced initial stress during irradiation, the recrystallisation which leads to a stress reduction and the stress reduction caused by the sample deflection itself.

\section{References}

[1] R. Salzer, A. Graff, F. Altmann, M. Simon Microsc. Microanal. 15 (Suppl. 2) (2009)

[2] S. Shim, H. Bei, M.K. Miller, G.M. Pharr, E.P. George Acta Materialia 57 (2009) 503-510

[3] F. Elfallagh \& B. J. Inkson J. Microsc. Vol. 230 Pt. 2 (2008) $240-251$

[4] L. Pastewka, R. Salzer Nucl. Instr. and Meth. B 267 (2009) 3072-3075 


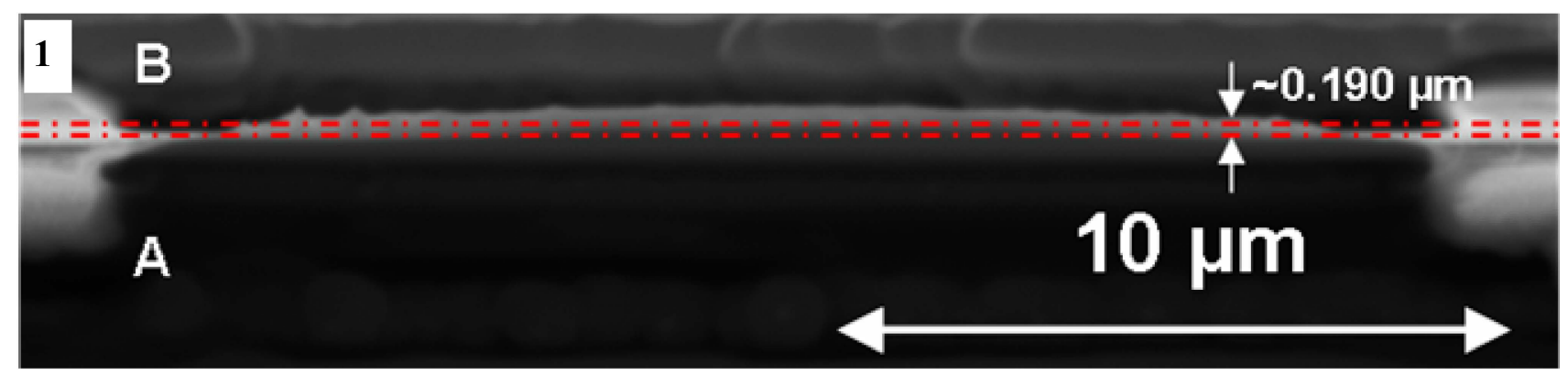

FIG. 1. SEM image of a whilst FIB preparation warped TEM sample, whereas side A of this sample is low voltage polished $\left(5 \mathrm{keV} \mathrm{Ga}^{+}\right)$and side $\mathrm{B}$ is milled with $30 \mathrm{keV} \mathrm{Ga}^{+}$
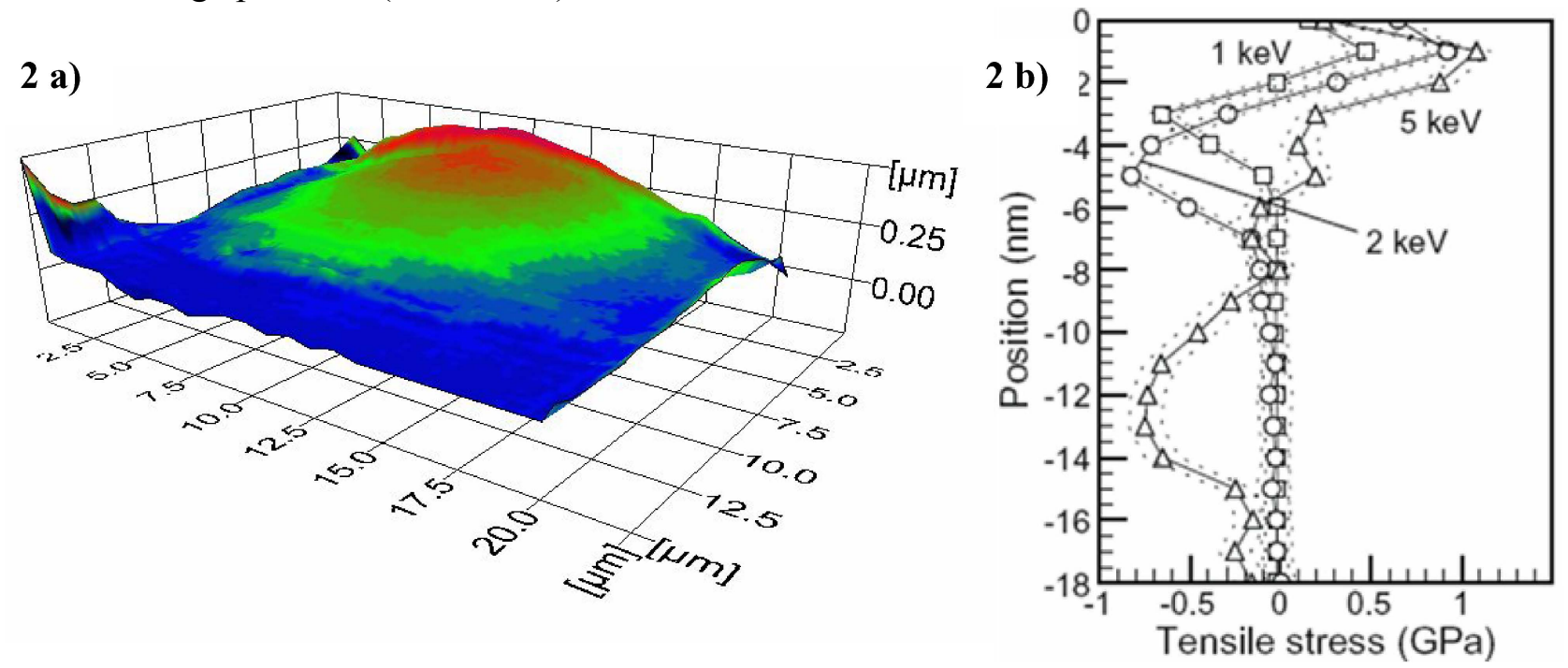

FIG. 2 a). 3D reconstruction of a LSM topographic image of an under FIB irradiation warped TEM sample. b) Results of MD calculations of the depth dependent stress value in a silicon surface for different low voltage $\mathrm{Ga}^{+}$irradiations, taken from [4]

3

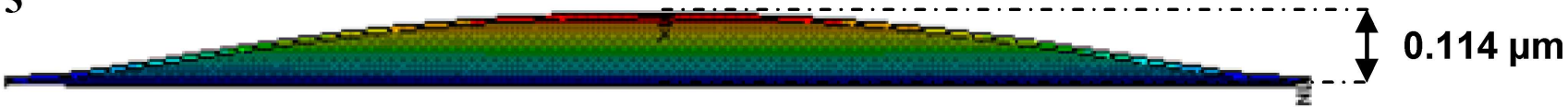

FIG. 3 FE model of a silicon TEM sample with a similar geometry as in FIG 1 and an extrapolated stress distribution for $30 \mathrm{keV}$ irradiation energy basing on $2 \mathrm{~b}$ ). 